'Instituto de Terapéutica Experimental y Clínica, Departamento de Fisiología, Centro Universitario de Ciencias de la Salud, Universidad de Guadalajara. ${ }^{2}$ Hospital Civil de Guadalajara "Fray Antonio Alcalde" Guadalajara Jalisco, México. ${ }^{\mathrm{a} M D}$

${ }^{\mathrm{b}} \mathrm{PhD}$.

Recibido el 12 de marzo de 2013, aceptado el $12 \mathrm{de}$ septiembre de 2013

Correspondencia a: Leonel García Benavides M.D., PhD.

Instituto de Terapéutica Experimental y Clínica, Departamento de Fisiología, CUCS, U de G. Sierra Mojada 950, edificio $P_{,} 1^{\circ}$ piso

Colonia Independencia, CP 44340

Guadalajara, Jalisco, México

Teléfono: (52) (33) 10585200

ext. 33659,33660

Fax: (52) (33) 36173499

E-mail:drleonelgb@ hotmail.com

\section{Triada mortal en pacientes politraumatizados, relación con mortalidad y severidad}

\author{
MARÍA GONZÁLEZ BALVERDE ${ }^{2, a}$, ERNESTO J. RAMÍREZ LIZARDO ${ }^{1, \mathrm{~b}}$, \\ ERNESTO G. CARDONA MUÑOZ ${ }^{1, b}$, SYLVIA E. TOTSUKA SUTTO ${ }^{1, b}$, \\ LEONEL GARCÍA BENAVIDES ${ }^{1,2, b}$

\section{Prognostic value of the lethal triad among patients with multiple trauma}

\begin{abstract}
Background: Patients who have suffered multiple traumatic injuries, have a serious risk for death. Hypothermia, acidosis and coagulopathy are three complications in these patients, whose presence is known as lethal triad and indicates bad prognosis. Aim: To determine if the lethal triad in multiple trauma patients is associated with higher mortality and Injury Score Severity (ISS). Material and Methods: One hundred multiple trauma patients aged 26 to 56 years (90 males), admitted to an emergency room, were studied. Body temperature, prothrombin time, partial thromboplastin time, platelet count and blood gases were determined on admission. Results: Twenty six patients had the lethal triad and 15\% died in the emergency room within the first 6 hours. No death was recorded among the 74 patients without the lethal triad. The mean ISS among patients with and without the lethal triad was 31.7 and 25.6, respectively $(p<0.05)$. Conclusions: The presence of the lethal triad among patients with multiple trauma is associated with a higher mortality and ISS.

(Rev Med Chile 2013; 141: 1420-1426)

Key words: Acidosis; Coagulation protein disorders; Hypothermia; Mortality; Multiple trauma.
\end{abstract}

S egún la Organización Mundial de la Salud fallecen cinco millones de personas anualmente a causa del trauma y casi $50 \%$ de ellas en edades entre 15 y 44 años. En México no se tienen estadísticas sobre traumatismos, éstas se encuentran englobadas en el rubro de accidentes. Los accidentes ocupan el tercer lugar de morbilidad y mortalidad a nivel nacional. Los accidentes de tráfico representan el mayor porcentaje causal, seguidos por lesiones deportivas, ahogamientos y quemaduras, con destacadas diferencias según el grupo de edad y sexo. Los traumatismos se clasifican de acuerdo a los órganos involucrados en: torácico, craneoencefálico, músculo esquelético, abdominal y en $2 / 3$ partes son mixtos. El paciente politraumatizado es definido como aquel individuo que presenta dos o más lesiones traumáticas graves ya sean periféricas o viscerales, que conllevan una repercusión res- piratoria o circulatoria y ponen en riesgo la vida. Los traumatismos están dentro de las cinco principales causas de morbilidad y mortalidad a nivel mundial, las lesiones del sistema nervioso central y la hemorragia son sus principales contribuyentes deletéreos $^{1}$. La acidosis, la hipotermia y la coagulopatía ${ }^{2}$ denominada la "triada mortal" se desarrolla como consecuencia de los cambios metabólicos inducidos por el politraumatismo.

Baker en 1974 creó un método para describir al paciente con múltiples daños y evaluar la emergencia, llamado ISS (Injury Severity Score) ${ }^{3}$. La puntuación es la suma de las calificaciones más altas de las tres regiones corporales más afectadas, obteniendo un índice crítico. Tornetta resalta claramente la importancia del resultado del ISS como factor pronóstico de mortalidad; una calificación del 0 a 18 representa una mortalidad menor a 5\%, 
Triada mortal en politraumatismo - M. González Balverde et al

de 19 a 30 la mortalidad es de $30 \%$, y más de 30 puntos de $45 \%{ }^{4}$.

Los pacientes politraumatizados son difíciles de valorar, diagnosticar y tratar, ya que presentan un riesgo vital elevado, por lo que se requiere un diagnóstico y tratamiento rápido, complejo y multidisciplinario; mediante pasos consecutivos y ordenados basados en la condición de cada paciente. El objetivo del trabajo es comparar si la presencia de la triada mortal en los pacientes politraumatizados que llegan al servicio de Urgencias Adultos del Hospital Civil de Guadalajara "Fray Antonio Alcalde" se asocia a mayor mortalidad y severidad del daño medido por la escala de ISS, lo que podría ser indicador de la eficacia y oportunidad con que se han implementado las estrategias terapéuticas.

\section{Material y Métodos}

Se diseñó un estudio observacional analítico, se incluyeron 100 pacientes politraumatizados que llegaron al servicio de Urgencias Adultos del Hospital Civil de Guadalajara "Fray Antonio Alcalde" con y sin la presencia de la triada mortal; a su ingreso se les tomaron las siguientes mediciones:

$p H$ : Se tomó muestra de sangre arterial para gasometría, procesada mediante procedimientos automatizados estándar, se determinó acidosis si el paciente presentaba resultados de $\mathrm{pH}$ menor a 7,35 y/o un bicarbonato menor a $21 \mathrm{mEq} / \mathrm{L}$.

Temperatura: Se tomó mediante termómetro digital óptico y la hipotermia fue documentada si era $\leq 36^{\circ}$.

Tiempo de coagulación: A todos los pacientes se les tomaron muestras de sangre para tiempo de protrombina (TP) y tiempo de tromboplastina parcial (TTP) procesadas mediante procedimientos automatizados estándar; para el diagnóstico de coagulopatía se consideró un TP mayor a 14,2 seg, un TTP superior a 38,4 seg y/o una trombocitopenia menor a $150,000 / \mathrm{uL}$.

Se calculó su ISS como aparece en la Tabla 1.

Tabla 1. Parámetros para evaluar severidad de la lesión (ISS) (injury score severity)

\begin{tabular}{|c|c|}
\hline Respiratorio & $\begin{array}{l}\text { 1. Dolor torácico } \\
\text { 2. Contusión o fractura simple } \\
\text { 3. Fractura de la primera costilla o múltiple, hemotórax o neumotórax } \\
\text { 4. Herida abierta, neumotórax a tensión, contusión pulmonar unilateral } \\
\text { 5. Insuficiencia respiratoria aguda, aspiración, contusión pulmonar bilateral, laceración diafragmática }\end{array}$ \\
\hline Sistema nervioso & $\begin{array}{l}\text { 1. Trauma cráneo encefálico sin pérdida del estado de alerta } \\
\text { 2. Fractura craneal, pérdida de la conciencia, Glasgow de } 15 \\
\text { 3. Lesión cerebral, fractura craneal deprimida, fractura facial deprimida, Glasgow menor de } 15 \\
\text { 4. Pérdida de la conciencia, Glasgow de menos de } 6 \text {, fractura cervical con paraplejia } \\
\text { 5. Coma durante más de } 24 \text { h, fractura cervical con tetraplejia } \\
\text { 6. Coma, pupilas dilatadas y fijas }\end{array}$ \\
\hline Cardiovascular & $\begin{array}{l}\text { 1. Pérdida de sangre menor de } 10 \% \\
\text { 2. Pérdida de sangre de } 10 \text { a } 20 \% \text {, contusión miocárdica } \\
\text { 3. Pérdida de sangre de } 40 \text { a } 50 \% \text {, TAM menos de } 80 \mathrm{~mm} / \mathrm{hg} \\
\text { 4. Pérdida de sangre de } 40 \text { a } 50 \% \text {, coma, agitación } \\
\text { 5. Pérdida de sangre mayor de } 50 \% \text {, coma, paro cardiaco }\end{array}$ \\
\hline Abdominal & $\begin{array}{l}\text { 1. Sensibilidad moderada en abdomen, signos peritoneales } \\
\text { 2. Fractura de costilla de } 7 \text { a } 12 \text {, dolor abdominal moderado } \\
\text { 3. Una sola lesión, hígado, bazo, riñón, intestino, uréter o páncreas } \\
\text { 4. Dos lesiones } \\
\text { 5. Dos lesiones severas: por aplastamiento hepático, lesión vascular mayor }\end{array}$ \\
\hline Músculo-esquelético & $\begin{array}{l}\text { 1. Esguince o fractura sin afectar el hueso largo } \\
\text { 2. Fractura simple, húmero, clavícula, radio, cúbito, tibia y peroné } \\
\text { 3. Fractura múltiple, simple de fémur, pélvica estable, luxación mayor } \\
\text { 4. Dos fracturas mayores, compleja de fémur, pelvis inestable, amputación } \\
\text { 5. Dos fracturas severas: Fracturas mayores múltiples }\end{array}$ \\
\hline Piel & $\begin{array}{l}\text { 1. Quemaduras menores de } 5 \% \text {, laceraciones, abrasiones, contusiones } \\
\text { 2. Quemaduras de } 5 \text { a } 15 \% \text {, contusiones extensas, avulsiones } \\
\text { 3. Quemaduras de } 15 \text { a } 30 \% \text {, avulsiones severas } \\
\text { 4. Quemaduras de } 30 \text { a } 45 \% \\
\text { 5. Quemaduras de } 45 \text { a } 60 \% \\
\text { 6. Quemaduras de más de } 60 \%\end{array}$ \\
\hline
\end{tabular}

El resultado se obtiene sumando el cuadrado de las calificaciones más altas de las 3 regiones corporales más afectadas. 
El trabajo cumple con las normas internacionales y fue revisado y aprobado por el comité de ética del Instituto de Terapéutica Experimental y Clínica del CUCS de la U de G.

Estadísticamente practicamos una serie de pruebas de asociación a cada grupo, utilizando la prueba exacta de Fisher, (mixto, tórax, craneoencefálico $(\mathrm{CE})$, cardiovascular $(\mathrm{CV})$, músculo esqueletico (ME), abdominal (abd) incluyendo la presencia o la ausencia de la triada mortal con el resultado de defunción.

Tabla 2. Población de estudio

\begin{tabular}{|lc|}
\hline & Valor \pm DE \\
\hline Total de casos & 100 \\
\hline Edad promedio (DS) & $28,8 \pm 11,89$ \\
\hline Género f/m & $90 / 10$ \\
\hline Aliento alcohólico & 44 \\
\hline Escala de Glasgow & $11,8 \pm 4,12$ \\
Índice de severidad de la lesión (ISS) & $27 \pm 10$ \\
Presencia de la triada mortal & 26 \\
Muerte & 4 \\
\hline
\end{tabular}

\section{Resultados}

En la Tablas 2 y 3 se muestran los datos generales y la condición de acuerdo al tipo de traumatismo. La triada mortal se presentó en 26 casos, con una mortalidad de $4 \%$, de los cuales dos fallecieron a la hora y los otros dentro de las tres horas de su estancia en el servicio de urgencias.

En la Tabla 4 se muestra un comparativo entre los pacientes que presentaron la triada mortal $26 \%$ y los que no, 74\%, donde se aprecian condiciones muy similares respecto a la edad, aliento alcohólico y escala de coma de Glasgow. En los paciente que presentaron la triada mortal los puntajes de severidad de la lesión (ISS) fueron más altos; ISS $(31,7$ versus 25,6 con una $p<0,05)$. Es importante señalar que las únicas defunciones en el servicio de urgencias se presentaron en el grupo de pacientes que tuvo la triada mortal representando $15,4 \%$ de este grupo (Fisher $=0,005)$. La asociación de la triada mortal resulta estadísticamente significativa para trauma mixto y CE (Fisher $=0,008$ ), y en las otras dos donde hay positividad a la triada mortal (tórax y ME), el "n" fue tan pequeño que no se puede establecer la asociación.

Tabla 3. Condición de los pacientes en relación al tipo de traumatismo

\begin{tabular}{|c|c|c|c|c|c|c|}
\hline Traumatismo & $\begin{array}{c}\text { Mixto } \\
\text { Valor } \pm \text { DE }\end{array}$ & $\begin{array}{c}\text { TX } \\
\text { Valor } \pm \text { DE }\end{array}$ & $\begin{array}{c}\text { CV } \\
\text { Valor } \pm \text { DE }\end{array}$ & $\begin{array}{c}\text { ME } \\
\text { Valor } \pm \text { DE }\end{array}$ & $\begin{array}{c}\text { CE } \\
\text { Valor } \pm \mathrm{DE}\end{array}$ & $\begin{array}{c}\text { ABD } \\
\text { Valor } \pm \mathrm{DE}\end{array}$ \\
\hline Total casos & 68 & 4 & 2 & 6 & 16 & 4 \\
\hline Edad & $29,7 \pm 11,5$ & $28 \pm 11,5$ & $16 \pm 1,4$ & $29,6 \pm 10,3$ & $29 \pm 14,6$ & $18 \pm 2,1$ \\
\hline Temperatura $\left({ }^{\circ} \mathrm{C}\right)$ & $36,2 \pm 0,8$ & $35,5 \pm 0,5$ & $36,9 \pm 0$ & $36,5 \pm 0,55$ & $37,5 \pm .7$ & $36,9 \pm 1,0$ \\
\hline $\begin{array}{l}\text { Presión arterial } \\
\text { media }\end{array}$ & $75,9 \pm 18,7$ & $54,4 \pm 20,6$ & $78,5 \pm 2,1$ & $59,5 \pm 21,0$ & $75,6 \pm 23,3$ & $71,4 \pm 5,6$ \\
\hline Glasgow & $12,4 \pm 3,7$ & $14,5 \pm 0,5$ & $14,5 \pm 0,7$ & $15 \pm 0$ & $6 \pm 1,6$ & $15 \pm 0$ \\
\hline ISS & $30,1 \pm 10,9$ & $21,5 \pm 4,0$ & $16,5 \pm 0,7$ & $23,3 \pm 6,7$ & $19 \pm 3,5$ & $25 \pm 8$ \\
\hline TP/SEG & $15,0 \pm 3,2$ & $15,8 \pm 2,5$ & $13,8 \pm 0,4$ & $15 \pm 1,0$ & $15,4 \pm 2,1$ & $12,7 \pm 1,2$ \\
\hline TPT/SEG & $30,4 \pm 15,7$ & $37,3 \pm 14,2$ & $24,4 \pm 0,7$ & $26,7 \pm 2,86$ & $27,3 \pm 4,4$ & $24,4 \pm 3,9$ \\
\hline INR & $1,37 \pm 0,48$ & $1,48 \pm 0,3$ & $0,93 \pm 0,4$ & $1,35 \pm 0,1$ & $1,37 \pm, 26$ & $1,06 \pm 0,1$ \\
\hline Acidosis & 46 & 4 & 0 & 2 & 10 & 2 \\
\hline Hipotermia & 48 & 4 & 0 & 2 & 4 & 2 \\
\hline Coagulopatía & 22 & 2 & 0 & 2 & 4 & 0 \\
\hline Triada mortal & 20 & 2 & 0 & 2 & 2 & 0 \\
\hline Muerte & 4 & 0 & 0 & 0 & 2 & 0 \\
\hline
\end{tabular}

TX: Tórax, CV: Cardiovascular, ME: Músculo esquelético, CE: Craneoencefálico, ABD: Abdominal. ISS: Índice de severidad de la lesión, TP: Tiempo de protrombina, TPT: Tiempo parcial de tromboplastina, INR: Razón Internacional normalizada. 
Triada mortal en politraumatismo - M. González Balverde et al

Tabla 4. Diferencias entre pacientes con y sin triada mortal

\begin{tabular}{|lccc|}
\hline Parámetro & Con triada mortal (Valor \pm DE) & Sin triada mortal (Valor \pm DE) & p \\
\hline Total de pacientes & 26 & 74 & 0,82 \\
\hline Edad & $29 \pm 11,4$ & $28 \pm 13,3$ & 0,76 \\
\hline Género m/f & $92 / 8$ & $89 / 11$ & 0,79 \\
\hline Aliento alcohólico & 46 & 43 & 0,64 \\
\hline Escala de Glasgow & $11,5 \pm 4,6$ & $11,9 \pm 3,9$ & $<0,01$ \\
ISS & $31,7 \pm 14,2$ & $25,6 \pm 8,2$ & $<0,01$ \\
\hline Presión arterial media & $65,1 \pm 15,3$ & $76,7 \pm 20,2$ & 0,62 \\
\hline Frecuencia cardiaca & $113 \pm 26,5$ & $115,4 \pm 20,6$ & $<0,05$ \\
\hline Frecuencia respiratoria & $27,3 \pm 10,4$ & $22,3 \pm 8,8$ & $<0,001$ \\
Temperatura $\left({ }^{\circ} \mathrm{C}\right)$ & $35,7 \pm 0,36$ & $36,6 \pm 1,2$ & $<0,001$ \\
TP & $18 \pm 3,2$ & $13,9 \pm 1,9$ & $<0,001$ \\
TPT & $41,9 \pm 21,2$ & $25,1 \pm 3,8$ & $<0,001$ \\
\hline INR & $1,8 \pm 0,5$ & $1,2 \pm 0,2$ & $<0,001$ \\
\hline Mortalidad $(\%)$ & 15,4 & 0 & \\
\hline
\end{tabular}

ISS: Índice de severidad de la lesión, TP: Tiempo de protrombina, TPT: Tiempo parcial de tromboplastina, INR: Razón Internacional normalizada.

\section{Discusión}

El servicio de Urgencias del Hospital Civil de Guadalajara recibe pacientes provenientes de la región occidente del país, los pacientes politraumatizados constituyen una de las principales causas de muerte, $33 \%$ de ellas ocurren en la sala de urgencias y son pacientes que por su gravedad requieren manejo multidisciplinario ${ }^{5}$. La principal complicación que provoca la muerte al paciente politraumatizado es la hemorragia; la coagulopatía secundaria al daño traumático se ha asociado al peor pronóstico y muerte ${ }^{6}$. El Colegio Americano de Cirujanos recomienda la reanimación agresiva basándose en modelos experimentales de los años cincuenta que incluyen: restitución del volumen sanguíneo con una relación 3:1 y el mantenimiento de la presión arterial dentro de los valores normales hasta el control de la hemorragia ${ }^{7}$. La hemorragia grave secundaria a trauma induce una respuesta inmunometabólica, inflamatoria y hemodinámica que lleva a hipoperfusión tisular, estado conocido como choque hemorrágico. Estos complejos mecanismos fisiopatológicos producen severas anormalidades metabólicas, las más notables son la coincidencia de acidosis, hipotermia y coagulopatía ${ }^{8}$. Estas condiciones si no logran ser previstas y controladas adecuadamente, llevarán rápidamente al paciente a la muerte. Nuestro estudio muestra que los pacientes que presentan la triada mortal tuvieron mayor mortalidad, ésta genera un círculo vicioso que se retroalimenta positivamente, por lo cual debemos planear el tratamiento del paciente politraumatizado, con el objetivo de lograr una mayor supervivencia, implementando pasos consecutivos y ordenados, basados en la tolerancia fisiológica del paciente. Estos eventos hace una década no se consideraban en la reanimación del paciente con trauma grave9.

En Estados Unidos de Norteamérica hay $30 \mathrm{mil}$ defunciones anuales por hemorragia aguda postraumática, la mitad de éstas en forma inmediata posterior a la lesión debido a ruptura de grandes vasos y el resto dentro de las $12 \mathrm{~h}$. Después de este período de tiempo las muertes son ocasionadas por choque compensado secundario a inadecuada reanimación, desarrollo de sepsis y disfunción orgánica múltiple ${ }^{10}$. La acidosis es una manifestación de trastorno metabólico, que utiliza una vía alterna en la producción de energía y puede reflejar hipovolemia, hipoxia, sepsis.

La acidosis metabólica en pacientes con poli- 
traumatismo ocurre primeramente como resultado de la producción de ácido láctico ${ }^{11,12} y$ ácido fosfórico, productos del metabolismo anaerobio causado por hipoperfusión orgánica ${ }^{13}$. La hemorragia produce una disminución de la oxigenación tisular que coincide con una disminución del gasto cardiaco y anemia. En pacientes con trauma torácico y contusión pulmonar puede haber un importante componente de acidemia respiratoria asociada a una inadecuada eliminación del $\mathrm{CO}_{2}{ }^{14}$.

Entre los efectos deletéreos de la acidosis se encuentran: depresión de la contractilidad miocárdica, disminución de la respuesta inotrópica a las catecolaminas, arritmias ventriculares, prolongación del tiempo de protrombina y del tiempo parcial de tromboplastina, disminución de la actividad del factor $V$ de la coagulación y coagulación intravascular diseminada (CID) por inactivación de varias enzimas de la cascada de coagulación.

La corrección de la acidosis requiere del control de la hemorragia y la optimización de la oxigenación tisular, lo que se logra inicialmente con la reposición de sangre y otros fluidos. Un paciente con hemorragia grave requiere una valoración acertada, tratamiento oportuno y pertinente lo que permitirá la conservación del volumen sanguíneo hacia los tejidos, para lo cual se requiere una reanimación cuidadosa y equilibrada con volumen, ya que existen evidencias que señalan que administrar grandes cantidades de líquidos antes del control de la hemorragia suele tener resultados adversos y que inclusive aumenta la mortalidad ${ }^{12,15}$. El ser humano es un ente homeotermo que mantiene una temperatura corporal constante y dentro de límites muy estrechos, entre $36,6+/-0,38^{\circ} \mathrm{C}$, a pesar de las amplias oscilaciones en la temperatura ambiental. La hipotermia se define cuando la temperatura central está por debajo de $35^{\circ} \mathrm{C}$ $\mathrm{y}$ es usualmente clasificada como; ligera (entre $32{ }^{\circ} \mathrm{C}$ y $35^{\circ} \mathrm{C}$ ), moderada (entre $32^{\circ} \mathrm{C}$ y $28^{\circ} \mathrm{C}$ ) y grave (por debajo de $28^{\circ} \mathrm{C}$ ). Esta clasificación que ha sido inicialmente diseñada para enfermos que han tenido una exposición accidental al frío, por ejemplo congelamientos, fue rediseñada para pacientes con traumatismo, debido a que éstos tienen mayores riesgos y complicaciones en estado de hipotermia, especialmente en aquellos con choque hemorrágico y se ha redefinido de la siguiente manera: ligera entre $36^{\circ} \mathrm{C}$ y $34^{\circ} \mathrm{C}$, moderada, entre $32{ }^{\circ} \mathrm{C}$ y $34^{\circ} \mathrm{C}$ y grave (por debajo de $\left.32{ }^{\circ} \mathrm{C}\right)^{13,16}$. Las causas de la hipotermia en el trauma son nu- merosas: la disminución de la oxigenación tisular y la consiguiente disminución de la producción de calor secundarias al shock hipovolémico, la restitución masiva de líquidos, sangre y derivados mantenidos en refrigeración, la exposición del paciente a bajas temperaturas tanto en la fase pre hospitalaria como hospitalaria (durante el examen físico con el enfermo totalmente expuesto), la inmovilización del paciente, la humedad de la ropa del paciente, la temperatura ambiente en las unidades de atención y tratamiento, los traumas de sistema nerviosos central (SNC) que pueden empeorar la termorregulación ${ }^{13}$. Los efectos sistémicos de la hipotermia son numerosos, entre ellos: depresión cardiaca por disminución de la frecuencia y del gasto cardiaco, aumento de la resistencia vascular sistémica, arritmias, disminución de la frecuencia de filtración glomerular y el empeoramiento de la absorción del sodio, disminución de la distensibilidad pulmonar, acidosis metabólica, depresión del sistema nervioso central, desviación de la curva de disociación de la hemoglobina por el oxígeno y en consecuencia hipoxia tisular, coagulopatía por el incremento de la actividad fibrinolítica, disminución de la actividad plaquetaria aún con adecuada reposición de estas; incremento del tiempo de protrombina y del tiempo parcial de tromboplastina; secuestro plaquetario en la circulación portal; inhibición de numerosas enzimas tanto de la vía intrínseca como de la vía extrínseca de la coagulación; disminución de la producción de tromboxano B2 con la consiguiente disminución de la agregación plaquetaria $^{15,17}$.

Aunque también han sido descritos efectos protectores de la hipotermia ${ }^{18,19}$, en nuestra opinión, coincidimos en que se trata de una complicación que agrava seriamente a los pacientes, pues produce lesiones secundarias sistémicas en pacientes con traumatismo grave; eleva la morbilidad y la mortalidad de los pacientes, al enrolarse en el círculo vicioso con las acidosis y la coagulopatía.

La coagulopatía es definida como la imposibilidad de la sangre de mantener un estado normal de coagulación, resultado de una depleción, dilución o inactivación de los factores de la coagulación ${ }^{20}$. Los valores que la definen es el tiempo de protrombina mayor de 14,2 seg o un tiempo parcial de tromboplastina superior 38,4 seg y con una trombocitopenia menor de 150,000/uL. Lapointe enmarca la coagulopatía grave como un tiempo 
de protrombina y parcial de tromboplastina dos veces mayor que el de los controles de laboratorio. La incidencia de coagulopatía en pacientes traumatizados es un predictor importante de mortalidad $^{21}$. Es frecuente que después de una reanimación agresiva con líquidos endovenosos $\mathrm{y}$ hemoderivados, se produzca un sangrado persistente en áreas lesionadas y no lesionadas, el cual conlleva inevitablemente a la muerte si no es posible controlarlo; esto se conoce como la coagulopatía del trauma. Se ha señalado que el resultado de la exanguinación y su tratamiento exponen a los pacientes a la coagulopatía, cuyo tratamiento incluye la reposición tanto de los elementos funcionales o elementos formes. El fibrinógeno es el primer factor que se afecta ${ }^{22}$ y puede alcanzar valores críticos inferiores a $1 \mathrm{~g} / \mathrm{L}$ cuando se pierde alrededor de 1,5 veces el volumen sanguíneo, le sigue la disminución de la actividad de otros factores de la coagulación cuando la volemia disminuye 2 veces aproximadamente. En nuestros resultados los pacientes fallecidos presentaron coagulopatía.

El trabajo publicado por Tornetta en 1999 hace alusión a la mortalidad según la escala de ISS $^{4}$, a mayor puntaje mayor índice de decesos, similar a lo observado en nuestro estudio, en el grupo que tenía 30 o más puntos presentaron mayor mortalidad. Sin embargo, existen condiciones que no se reflejan en la valoración por ISS e impactan en la mortalidad de los pacientes como; la presencia de lesiones graves pero no complejas, que involucran un solo aparato o sistema como el TCE, el tiempo transcurrido en que se inicia la reanimación, las maniobras de reanimación y el retraso en que se implementa el tratamiento definitivo y que tienen una mayor asociación con la triada mortal.

\section{Conclusiones}

Existe relación entre la presencia de la triada mortal con la severidad de la lesión valorada por el índice de severidad de la lesión, (ISS) ya que los pacientes que presentaron la triada mortal tenían puntajes de 30 o más de ISS.

La presencia de la triada mortal se asoció a una mayor mortalidad con independencia del tipo de traumatismo. En el análisis por grupos; el trauma mixto y craneoencefálico son los más frecuentes y la presencia de la triada mortal se asociaron a mayor mortalidad, por lo que la triada mortal podría ser un indicador de la magnitud de la lesión y de la oportunidad y pertinencia de las maniobras de reanimación implementadas.

\section{Referencias}

1. Asensio JA, Petrone P, O'Shanahan G, Kuncir EJ. Managing exsanguination: what we know about damage control/bailout is not enough. Proceedings 2003; 16 : 294-6.

2. Schreiber MA. Damage control surgery. Crit Care Clin 2004; 20 (1): 101-18.

3. Baker SP, O'Neill B, Haddon W Jr, Long WB. The injury severity score: a method for describing patients with multiple injuries and evaluating emergency care. J Trauma 1974; 14 (3): 187-96.

4. Afuwape OO, Ogunlade SO, Alonge T, Ayorinde OR. An audit of deaths in the emergency room in the University College Hospital Ibadan Niger. J Clin Pract 2009; 12 (2): 138-40.

5. Davenport R. Pathogenesis of acute traumatic coagulopathy. Transfusion 2013; 53 Suppl 1: 23S-7S.

6. Hameed S, Aird W, Cohn M. Oxygen delivery. Crit Care Med 2003; 31: 658.

7. Rotondo MF, Schwab CW, McGonigal MD, Phillip OR III, Fruchterman TM, Kauder DR, Latenser BA, Angood PA. "Damage Control”: An approach for improved survival in exsanguinating penetrating abdominal injury. J Trauma 1993; 35: 375-83.

8. Shapiro MB, Jenkins DH, Schwab CW, Rotondo MF. Damage control: collective review. J Trauma 2000; 49 (5): 969-78.

9. Dutton RP. Low-pressure resuscitation from hemorrhagic shock. Intern Anesthes Clin 2003; 40: 19-30.

10. De Waele JJ, Vermassen FE. Coagulopathy, hypothermia and acidosis in trauma patients: the rationale for damage control surgery. Acta Chir Belg 2002; 102 (5): 313-6.

11. Lapointe LA, Von Rueden KT. Coagulopathies in trauma patients. AACN 2002; 13 (2): 192-203.

12. Revell M, Porter K, Greaves I. Fluid resuscitation in prehospital trauma care: a consensus view. Emerg Med 2002; 19: 494-8.

13. Schreiber MA. Damage control surgery. Crit Care Clin 2004; 20 (1): 101-18.

14. Graham CA, Parke TRJ. Critical Care in the emergency department: shock and circulatory support. Emerg Med 2005; 22: 17-21.

15. De Waele JJ, Vermassen FE. Coagulopathy, hypothermia and acidosis in trauma patients: the rationale for damage control surgery. Acta Chir Belg 2002; 102 (5): 313-6. 
16. Parr MJ, Alabdi T. Damage control surgery and intensive care. Injury 2004; 35 (7): 713-22.

17. Childs EW, Udobi KF, Hunter FA. Hypothermia reduces microvascular permeability and reactive oxygen species expression after hemorrhagic shock. J Trauma 2005; 58 (2): 271-7.

18. Tisherman SA. Hypothermia and Injury. Curr Crit Care 2004 (10): 512-19.

19. Cosgriff N, Moore EE, Azuaya A, Kenny M, Burch JM, Galloway B. Predictive Life-threatening coagulopathy in the massively transfused trauma patient: hypothermia and acidosis revised. J trauma 1997; 42: 857-62.

20. Lapointe LA, Von Rueden KT. Coagulopathies in trauma patients. AACN 2002; 13 (2): 192-203.

21. Spahn DR, Rossaint R. Coagulopathy and blood component transfusion in trauma. British Journal of Anaesthesia 2005; 95 (2): 130-9.

22. Tornetta P, Mostafari H, Riena J, Turen C, Reiner B, Levine $\mathrm{R}$, et al. Morbidity and mortality in elderly trauma patients. J Trauma 1999; 46 (4): 702-6. 